\title{
Characterization of dextrin hydrogels by FTIR spectroscopy and solid state NMR spectroscopy
}

\author{
Helga Garcia a, António S. Barros ${ }^{b}$, Catarina Gonçalves ${ }^{c}$, Francisco M. Gama ${ }^{c}$, Ana M. Gil a,* \\ a CICECO, Department of Chemistry, Campus Universitário de Santiago, University of Aveiro, 3810-193 Aveiro, Portugal \\ ${ }^{\mathrm{b}}$ QOPNAA, Department of Chemistry, Campus Universitário de Santiago, University of Aveiro, 3810-193 Aveiro, Portugal \\ ${ }^{\mathrm{c}}$ IBB - Institute for Biotechnology and Bioengineering, Centre of Biological Engineering, Universidade do Minho, Campus de Gualtar, $4710-057$ Braga, Portugal
}

\section{A R T I C L E I N F O}

\section{Article history:}

Received 26 March 2008

Received in revised form 29 April 2008

Accepted 6 May 2008

Available online 28 May 2008

\section{Keywords:}

Dextrin

Hydrogel

FTIR

CP/MAS

PLS

2D-COS

\begin{abstract}
A B S T R A C T
Fourier transform infrared (FTIR) and ${ }^{13} \mathrm{C}$ solid state nuclear magnetic resonance (NMR) spectroscopy were used to study dextrin structural changes occurring upon hydrogel formation by vinyl acrylate (VA) grafting and subsequent free radical polymerization. The degrees of VA substitution (DS) and polymerization (DP) were quantified up to 40\%VA by FTIR intensity measurements and partial least squares (PLS)/FTIR, the latter being a faster and less error-prone method. Above 40\%VA, both parameters are underestimated by FTIR. A spin counting NMR experiment showed high carbon observabilities for hydrogels and improved PLS/NMR models were achieved for DS and DP determination. Alternative NMR integration methods are hindered by the broad VA peaks and need for area correction, due to their $\mathrm{CP}$ dynamics. NMR changes in $\mathrm{C} 1$ profile showed that a single helical conformation predominates at lower \%VA, being replaced by disordered conformations as \% VA increases. Furthermore, a correlation FTIR/NMR study indicated that ring conformations are significantly affected in hydrogels, compared to unpolymerized dextrin.
\end{abstract}

(c) 2008 Elsevier Ltd. All rights reserved.

\section{Introduction}

Hydrogels is a term increasingly used to classify highly hydrated polymeric networks, usually composed of hydrophilic polymer chains [1]. Such networks are held by either chemical or physical cross-links between polymer chains. The increasing interest in hydrogel production is justified by their great potential in several areas of medical research [2], including drug delivery systems [3], scaffold materials for cell and tissue engineering [4]. Hydrogels may be obtained from natural sources or prepared entirely through synthetic pathways [5] and efforts are continuously being made to develop new hydrogel materials based on the natural abundance, biocompatibility, biodegradability, ease of derivatization and modification of some polymers. Starchbased polymers like dextrin and dextran are examples of

\footnotetext{
* Corresponding author. Tel.: +351 234 370707; fax: +351 234370084 E-mail address: agil@ua.pt (A.M. Gil).
}

natural hydrophilic polymers with great potential in this area [6,7] and several reports may be found on the methods, both chemical and enzymatic, for their derivatization into hydrogel structures [8-10].

Dextrin results from the partial hydrolysis of starch and is exclusively composed of $\alpha(1 \rightarrow 4)$ linked D-glucose residues, contrary to dextran which is a bacteria polysaccharide containing a high percentage of $\alpha(1 \rightarrow 6)$ branching points. Being widely used in food industry, textiles and other areas due to its biocompatibility and degradability, dextrin offers great potential for hydrogel production. One way of using dextrin to produce hydrogels has been described as involving a first modification with vinyl acrylate (VA), using either a chemical or an enzymatic approach, followed by covalent cross-linking through VA residues through free radical polymerization in solution [11]. Although the products of dextrin transesterification with VA are water-soluble thus enabling analysis through a wide range of methods, including ${ }^{1} \mathrm{H}$ nuclear magnetic resonance (NMR) spectroscopy 
[12], the polymerized systems (or hydrogels) are often water insoluble. In tandem with X-ray diffraction and microscopic characterization of the materials, the characterization of hydrogels at the molecular level may be carried out by solid state analytical methods such as Fourier transform infrared (FTIR) spectroscopy or solid state nuclear magnetic resonance (NMR) spectroscopy. FTIR spectroscopy has been employed before to characterize dextran-based systems [9] and FTIR intensity ratios were used to quantify their degree of substitution (DS) with VA and the extent of polymerization of the resulting hydrogels.

This work describes how FTIR spectroscopy may be further explored for quantitation purposes and to obtain improved insight into the structural modifications taking place in the dextrin network, by using partial least squares (PLS) regression to extract overall spectral information. The possibility of rapid quantitation of DS, in unpolymerized samples, and the degree of polymerization (DP), in hydrogels, using PLS/FTIR models is discussed. In addition, solid state ${ }^{13} \mathrm{C}$ cross polarization and magic angle spinning $(\mathrm{CP} /$ MAS) NMR is also explored for DS and DP quantitation as well as for characterization of the structural and molecular dynamic characteristics of the dextrin hydrogel samples. PLS/NMR models for quantitation of DS and DP are also developed and their applicability, compared to FTIR-based methods, is discussed. Finally, a bidimensional correlation method is used to correlate between FTIR and NMR data, as a function of DS and DP, in order to improve interpretation of both datasets for dextrin.

\section{Experimental section}

\subsection{Samples}

Two groups of dextrin samples were investigated in this study: (a) a group comprising unmodified dextrin and seven dextrin samples enzimatically substituted to different degrees (10\%, 14\%, 18\%, 21\%, 31\%, 38\% and $67 \%$ ) with reactive VA groups and (b) a group of polymerized hydrogel samples obtained from the samples in (a) using a free radical polymerization method described elsewhere [11]. Since the last two samples showed indications of being less porous and, therefore, less interesting as hydrogel materials, no additional samples were prepared for \%VA between $40 \%$ and 67\%. Fig. 1 shows a schematic representation of the modification steps employed. The extent of VA substitution was determined for samples in group (a) by solution state ${ }^{1} \mathrm{H}$ NMR spectroscopy [12], a reference method for DS determination in dextrin-like materials, the precise experimental conditions of which are described in Ref. [13]. Samples were found to contain $10-12 \% \mathrm{w} / \mathrm{w}$ humidity and no sample

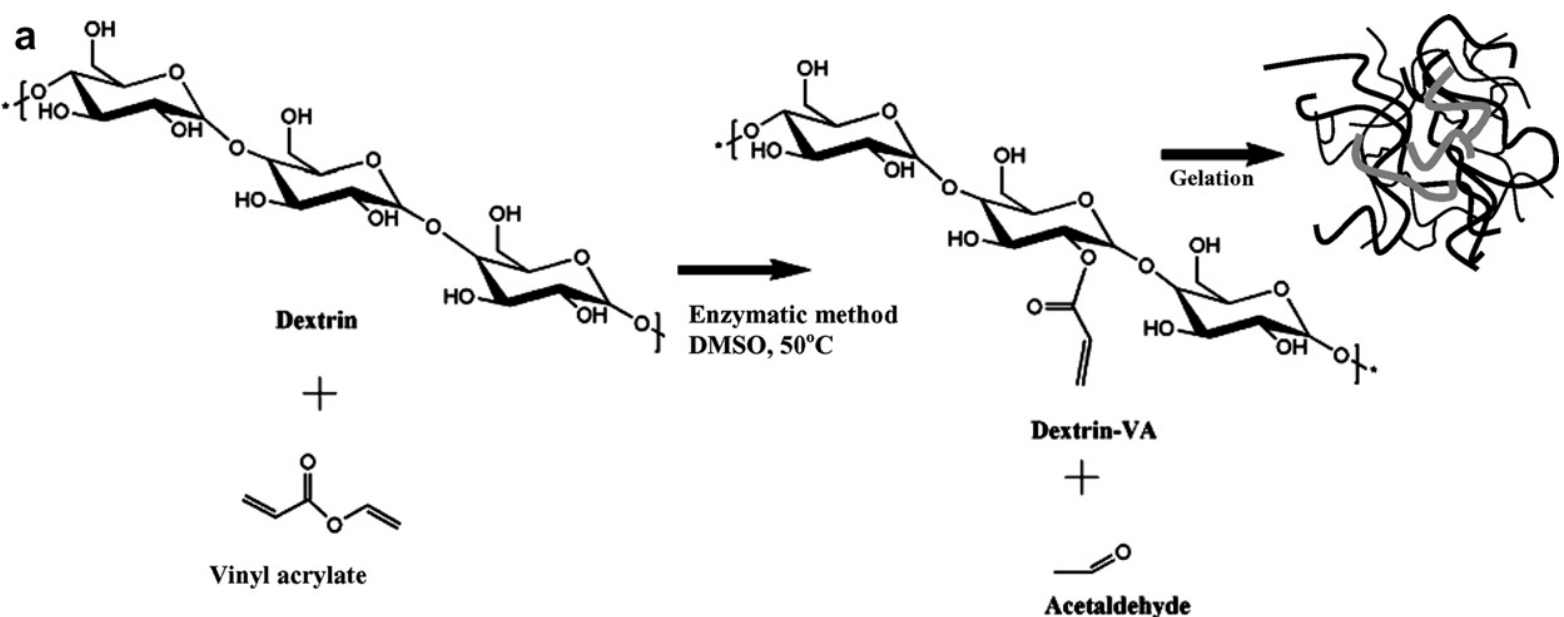

b

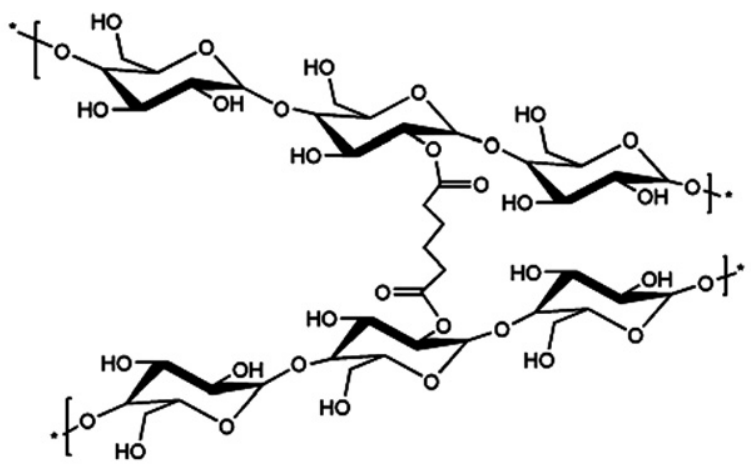

Dextrin-VA Hydrogel

Fig. 1. Schematic representation of: (a) synthesis of dextrin-VA and hydrogel networks and (b) hydrogel cross-links. 
drying was carried out before analysis. The glycine sample used for NMR spin counting experiments was purchased from Aldrich and used as received.

\subsection{Attenuated total reflectance (ATR) FTIR spectroscopy}

FTIR spectra of dextrin unpolymerized and polymerized (hydrogel) dextrin samples were collected on a Bruker IFS55 FTIR spectrometer, using a single reflectance ATR cell (Golden Gate, equipped with a diamond crystal). All data were recorded at room temperature, in the spectral range of $4000-700 \mathrm{~cm}^{-1}$, by accumulating 256 scans with a resolution of $8 \mathrm{~cm}^{-1}$. Five replica spectra were collected for each sample in order to evaluate experimental reproducibility. For discrete band intensity measurements, the average spectra were considered and baseline correction in the $800-600 \mathrm{~cm}^{-1}$ spectral region was carried out manually. For unpolymerized samples, DS was computed simply by taking the $I_{806} / I_{760}$ intensity ratio and, for polymerized samples, the extent of polymerization was evaluated using the following expression, where $I_{806} / I_{760}$ ratios before and after polymerization are compared:

$\%$ polymerization $=\frac{\left(\frac{I_{806}}{I_{760}}\right)_{\text {before }}-\left(\frac{I_{806}}{I_{760}}\right)_{\text {after }}}{\left(\frac{I_{808}}{I_{760}}\right)_{\text {before }}} \times 100$

\subsection{Solid state ${ }^{13} \mathrm{C} C P / M A S ~ N M R$ spectroscopy}

All ${ }^{13} \mathrm{C} \mathrm{CP} / \mathrm{MAS}$ spectra were recorded on a Bruker AVANCE-400 (DRX) NMR spectrometer operating at $400 \mathrm{MHz}$ for proton and $100 \mathrm{MHz}$ for carbon, using $7 \mathrm{~mm}$ $\mathrm{CP} / \mathrm{MAS}$ Bruker double-bearing probes. All samples were finely ground before packing in the rotors. Rotors were spun at rates $6 \mathrm{kHz}$ and the ${ }^{13} \mathrm{C} \mathrm{CP} / \mathrm{MAS}$ spectra were recorded using a proton $90^{\circ}$ pulse length of $4.0 \mu \mathrm{s}, 1 \mathrm{~ms}$ contact time and recycle time of $10 \mathrm{~s}$ for dextrin samples and $2 \mathrm{~s}$ for glycine, using the $5 \times \mathrm{T}_{1 \mathrm{H}}$ (proton longitudinal relaxation time) condition to ensure full proton relaxation between scans. $T_{1 \mathrm{H}}$ values were measured through a modification of the $\mathrm{CP} / \mathrm{MAS}$ experiment so as to include an inversionrecovery step on the proton channel and using interpulse delays in the $10 \mathrm{~ms}-10 \mathrm{~s}$ range. Relaxation $T_{\mathrm{CH}}$ and $T_{1} \rho_{\mathrm{H}}$ constants were measured by fitting the peak intensity curves resulting from variable contact time experiments with 18-24 different points in the $0.01-15 \mathrm{~ms}$ range. The intensity curve recorded as a function of contact time, $\tau$, takes the form:

$I(\tau)=I_{0} \times\left[\exp \left(-\frac{\tau}{T_{1 \rho \mathrm{H}}}\right)\right] \times\left[1-\exp \left(-\frac{\tau}{T_{\mathrm{CH}}}\right)\right]$

with $I_{0}$ representing the maximum signal intensity. For spin counting experiments, glycine was used as an external reference, instead of mixed with the polymer and thus rendering the sample unusable as well as posing the risk of uneven mixing and subsequent varying NMR sensitivity [14]. As required when an external reference compound is used, special care was taken to record the glycine spectrum in exactly the same experimental conditions as those of the samples, therefore, glycine and all dextrin samples were run within 2-3 days so as to minimize deviations in experimental settings such as probe tuning and HartmanHahn condition. Spin counting experiments were carried out for three selected hydrogels (with 20, 38 and 67\% VA) and, for each spectrum, the integrated intensity $I$ was divided by the sample mass $m$, giving $I^{*}(=I / m)$. The relationship [14]:

$I^{*}=k \times \% C \times C_{\text {obs }}$

was used, where \%C was determined by elemental analysis and constant $k$ determined by assuming carbon observability $\left(C_{\mathrm{obs}}\right)$ of $100 \%$ for glycine. $C_{\mathrm{obs}}$ is the carbon observability (in\%) obtained for each hydrogel sample. To evaluate the improvement of these $C_{\mathrm{obs}}$ values, the average $T_{1} \rho \mathrm{H}$ was measured for each hydrogel, through variable contact time experiments with improved precision (12-18 points) in the decaying part of the $\mathrm{CP}$ curve and used to correct spectral area according to $T_{1} \rho_{\mathrm{H}}$ differences, as described in Ref. [14].

\subsection{Partial least squares (PLS1) regression}

Partial least squares regression (PLS1) is a widely used method to perform data analysis in a vast range of applications [15-18]. In a multivariate regression context, the following equation applies:

$\mathbf{y}_{(n, 1)}=\mathbf{X}_{(n, m)} \mathbf{b}_{(m, 1)}+\mathbf{e}_{(n, 1)}$

where $n$ is the number of objects, $m$ is the number of variables, $y$ is the dependent response vector, $\mathbf{X}$ is the matrix of independent variables and the $\mathbf{e}$ vector is the variability not accounted by the regression model. The $\mathbf{b}$ vector estimation based on the PLS1 regression is given by:

$\mathbf{b}_{(m, 1)}=\mathbf{W}_{(m, k)}\left[\mathbf{P}_{(k, m)}^{T} \mathbf{W}_{(m, k)}\right]^{-1} \mathbf{c}_{(k, 1)}^{T}$

where $k$ is the number of latent variables, $\mathbf{W}$ the loadings weights, $\mathbf{P}$ the loadings $X$ and $\mathbf{c}$ the loadings $y$. An essential step in the PLS1 regression is the assessment of the model complexity, i.e. the number of latent variables that must be used in order to describe the relationship between the dependent responses (y) and the independent variables (X). Among the many approaches used to assess the number of latent variables, the cross-validation is one of the most frequently employed.

\subsection{Two dimensional correlation spectroscopy (2D-COS)}

Two dimensional correlation spectroscopy (2D-COS) is a spectral technique developed for evaluating 2-way data sets obtained when a sample is subject to an external sequential perturbation [19]. This perturbation-based 2D so called spectroscopy tool aims at sorting out complex or overlapped spectral features by spreading peaks along a second dimension $[20,21]$. The method detects in-phase (synchronous) and out-of-phase (asynchronous) correlations between spectral intensity variations that occur at different points in a spectral signal and that are stimulated by external perturbations [19,20,22,23].

The synchronous correlation intensity map, $\Phi_{(v 1, v 2)}$ represents the similarity between two separated spectral 
intensity variations, at $v 1$ and $v 2$, as a function of an external perturbation. In the case of hetero-spectral 2D-COS, the synchronous map represents synchronized changes of spectral features of two different bands ( $v 1$ and $v 2$ ) in different data sets, suggesting related origins for spectral variations.

\section{Results and discussion}

\subsection{FTIR spectroscopy}

Fig. 2a shows the ATR-FTIR spectra of original dextrin and some of the dextrin samples substituted to different degrees with reactive VA groups. In Fig. $2 b$, the ATR-FTIR spectra obtained for the corresponding hydrogel samples are shown. The spectrum of dextrin shows the typical starch-like FTIR profile and the main peaks arising from VA moieties are identified with arrows in both unpolymerized and polymerized samples. In unpolymerized dextrin samples, the two VA bands at $1713 \mathrm{~cm}^{-1}$ and $806 \mathrm{~cm}^{-1}$ arise, respectively, from the carbonyl group stretch and from the twisting of the acrylate double bond [9]. The two bands at 1612 and $1628 \mathrm{~cm}^{-1}$ should arise from VA double bonds stretch vibrations, overlapping with a band at $1635 \mathrm{~cm}^{-1}$ observed in the spectrum of original dextrin. The latter was found to arise from water, since it disappeared with sample drying. Other dextrin bands appear at 1408,1296 and $1192 \mathrm{~cm}^{-1}$, in the fingerprint region (Fig. 2).
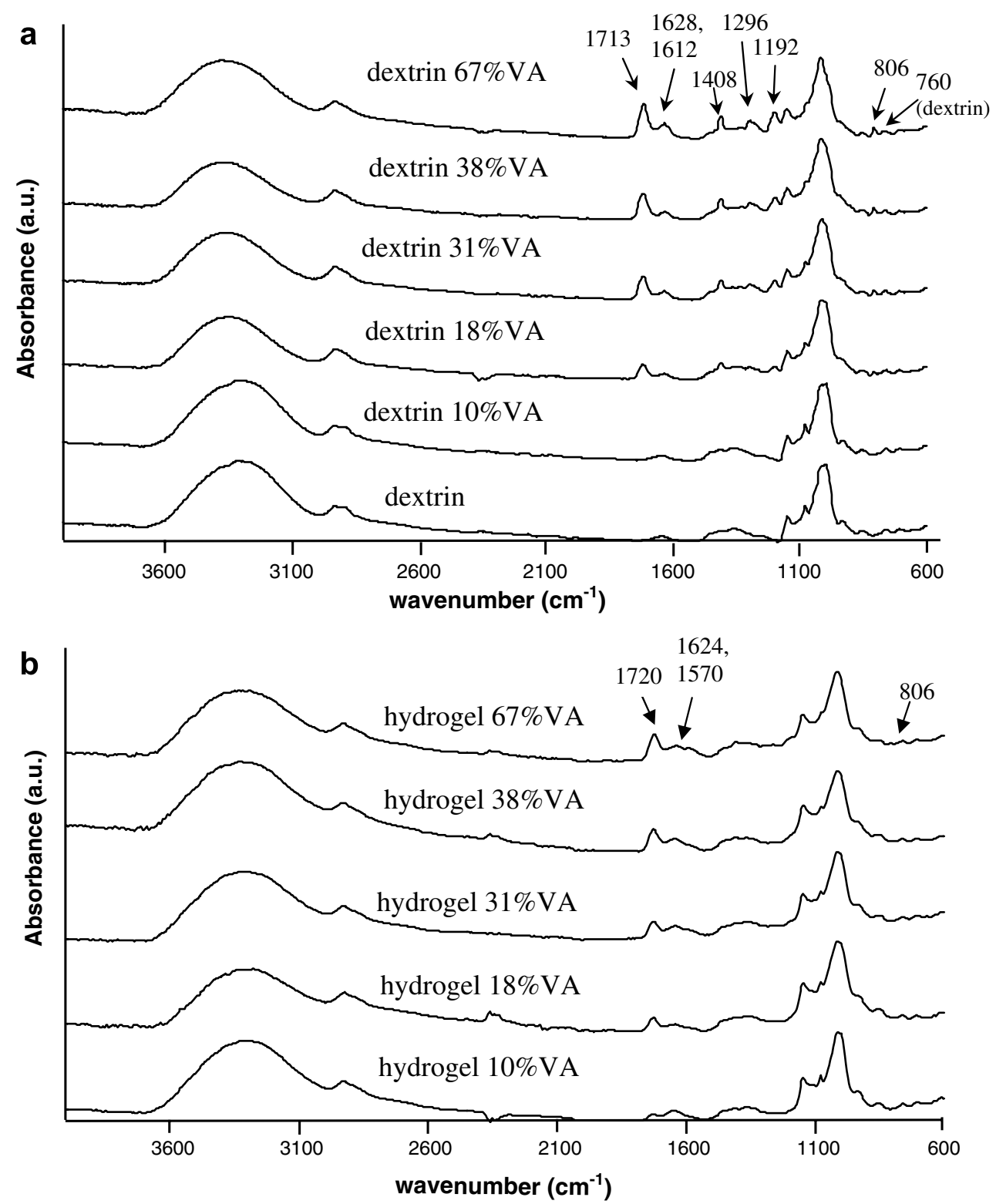

Fig. 2. ATR-FTIR spectra of: (a) original dextrin and dextrin substituted with 10,18, 31, 38 and $67 \%$ VA and (b) corresponding polymerized samples (hydrogels). With the exception of the $760 \mathrm{~cm}^{-1}$ band (dextrin), the peaks identified with arrows correspond to main VA bands. 
In the FTIR spectra of the hydrogels, the main changes upon polymerization are a slight broadening of the spectra and relative decreases at 806, 1408 and $1612-1628 \mathrm{~cm}^{-1}$, due to the decrease in the number of double bonds as cross links are formed. In addition, the $\mathrm{C}=\mathrm{O}$ stretch vibration (at $1713 \mathrm{~cm}^{-1}$ in unpolymerized samples) shifts to higher wavenumbers $\left(1720 \mathrm{~cm}^{-1}\right)$, an effect previously reported for dextran-based hydrogels [9] and which reflects the changes in the carbonyl environment upon the conversion of VA moieties into cross-links. The band at $1612 \mathrm{~cm}^{-1}$ is also seen to shift to $1570 \mathrm{~cm}^{-1}$ upon polymerization, suggesting a distinct environment for double bonds in residual unreacted VA residues.

Dextrin gives an FTIR band at $760 \mathrm{~cm}^{-1}$ which, in previous studies carried out on the similar system of dextran [9], has been seen not to be significantly affected by either VA substitution or polymerization. This band has, therefore, been often chosen as a reference band against which other peak variations may be compared [9]. The $I_{806} / I_{760}$ intensity ratio was hereby used to determine DS in unpolymerized samples, after application of baseline correction in the relevant spectral region. Fig. 3a shows the variation of this FTIR intensity ratio as a function of \% VA, as determined by ${ }^{1} \mathrm{H}$ NMR spectroscopy. A linear tendency is noted up to the dextrin-38\% VA sample, followed by a slight deviation from linearity defined by the last sample. This indicates that FTIR-based DS values above $c a$. 40\% VA may be underestimated relatively to ${ }^{1} \mathrm{H}$ NMR measurements. One possible reason is that structural changes occur in dextrin samples with higher \%VA, thus affecting dextrin FTIR bands more significantly, including the $760 \mathrm{~cm}^{-1}$ band, previ- ously assumed suitable as a reference band. PLS regression analysis was used in order to take into account changes across the whole FTIR spectrum, instead of only at 760 and $806 \mathrm{~cm}^{-1}$. Fig. 3b shows that a satisfactory correlation is found between the \%VA predicted from FTIR spectra and the actual value, however, the less than ideal $R^{2}(0.96)$ value reflects the same tendency to lower FTIR DS values for higher \%VA, compared to the reference NMR method. The b vectors corresponding to the PLS/FTIR model (Fig. 3c) confirm that spectral variations between samples arise mainly from the variation in VA content (positive VA peaks noted with arrows). However, additional changes do occur in the dextrin fingerprint region, reflecting the occurrence of structural changes in the polysaccharide structure as VA grafting is applied.

The extension of polymerization and other characteristics of the hydrogels are more challenging subjects due to the insolubility of the samples. The extent of polymerization in hydrogels, as evaluated by FTIR intensity measurements (see experimental), gives similar polymerization yields of $80-90 \%$ for all samples (Table 1). Considering the known percentages of grafted \%VA, the number of cross-linked VA residues per 100 residues of glucose may be easily calculated and taken as the degree of polymerization, DP (Table 1). Fig. 4a shows that a good linear dependence exists between the DP in the hydrogels and the percentage of initially grafted VA residues, meaning that, for this type of samples, a DP value may be estimated throughout the range studied, directly from the initial \%VA of the grafted material and assuming a linear dependence.
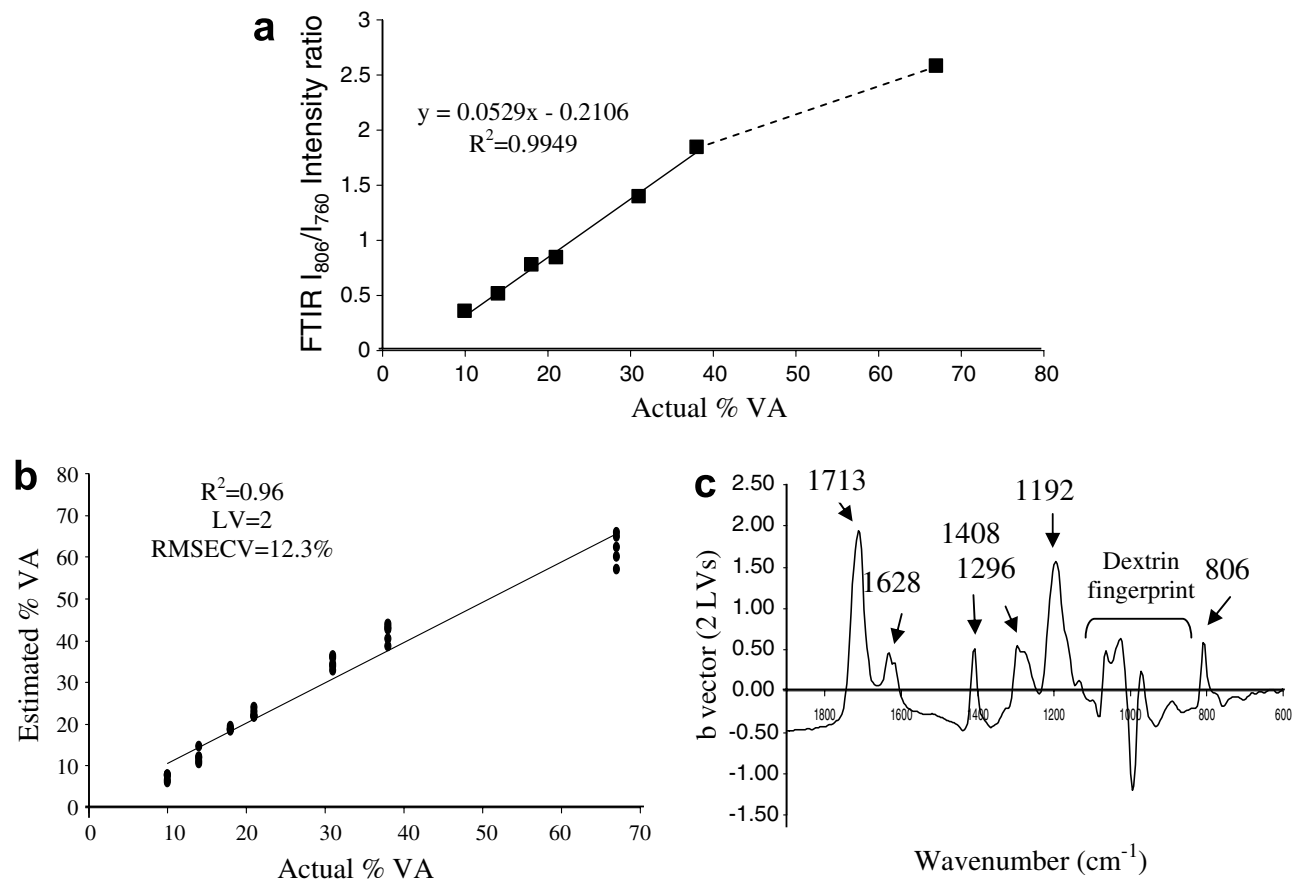

Fig. 3. (a) Graphical representation of the FTIR $I_{806} / I_{760}$ intensity ratio for unpolymerized dextrin samples, as a function of $\%$ VA as determined by ${ }^{1} \mathrm{H}$ NMR spectroscopy; PLS/FTIR, (b) relationship between actual and estimated \%VA and (c) b vector for unpolymerized samples. Peaks indicated with arrows arise from VA moieties. 
Table 1

FTIR intensity ratios for each hydrogel sample, extension of polymerization (\%) and corresponding degree of polymerization DP

\begin{tabular}{lllll}
\hline Hydrogel sample & $\begin{array}{l}\text { FTIR } I_{806} / I_{760} \\
\text { before polym. }\end{array}$ & $\begin{array}{l}\text { FTIR } I_{806} / I_{760} \\
\text { after polym. }\end{array}$ & $\begin{array}{l}\% \\
\text { Polymerization }\end{array}$ & DP $^{\mathrm{a}}$ \\
\hline Dextrin-VA 10\% & 0.349 & 0.0494 & 85.8 & 9 \\
Dextrin-VA 14\% & 0.498 & 0.0803 & 83.9 & 12 \\
Dextrin-VA 18\% & 0.773 & 0.1091 & 85.9 & 16 \\
Dextrin-VA 21\% & 0.843 & 0.0968 & 88.5 & 19 \\
Dextrin-VA 31\% & 1.396 & 0.137 & 90.2 & 28 \\
Dextrin-VA 38\% & 1.839 & 0.227 & 87.6 & 33 \\
Dextrin-VA 67\% & 2.578 & 0.455 & 82.4 & 55 \\
\hline
\end{tabular}

a Expressed in number of VA residues per 100 glucose residues.

PLS/FTIR analysis was performed on the hydrogels spectra, taking as reference the FTIR-based DP values calculated as described above and aiming at building a model for rapid evaluation of DP. The relationship plot between actual DP vs. estimated DP (Fig. 4b) shows that, again for the $67 \%$ VA hydrogel, relatively lower DP values are predicted by PLS, compared to the actual DP values. The corresponding b vector (Fig. 4c) is rather similar to that obtained for the unpolymerized samples (Fig. 3c), with positive VA bands explaining most spectral variation, together with some changes in the dextrin fingerprint region. Again, these confirm structural changes occurring in the polysaccharide, in tandem with the increase in VA cross-links, but the complex nature of the fingerprint FTIR region hinders further interpretation of these changes.

Therefore, although both intensity measurements and PLS/FTIR method underestimate DS and DP for dextrinbased samples with \%VA higher than ca. 40, they may be used for quantitation up to that VA content. PLS/FTIR is a faster approach compared to the intensity measurement method, avoiding error-prone methods such as baseline correction and manual intensity ratio measurements of weak bands such as the 806 and $760 \mathrm{~cm}^{-1}$ bands.

\subsection{Solid state NMR spectroscopy}

Fig. 5 shows the ${ }^{13} \mathrm{C} \mathrm{CP} / \mathrm{MAS}$ spectra of original dextrin, as well as of some unpolymerized (Fig. 5a) and polymerized (Fig. 5b) dextrin samples. In order to ensure full proton relaxation, all spectra were recorded using recycle delays between consecutive scans of $5 \times \mathrm{T}_{1 \mathrm{H}}$, based on the $T_{1 \mathrm{H}}$ values measured for a selection of samples (Table 2). Proton $T_{1 \mathrm{H}}$ relaxation times were shown not to depend significantly on VA content which means that rapid molecular motions (in the $\mathrm{MHz}$ timescale), to which $T_{1}$ (or longitudinal) relaxation is most sensitive, do not vary significantly between samples. The approximate common value of ca. $1 \mathrm{~s}$, for each sample, reflects the effective proton spin diffusion in all samples, due to their solid proton-rich nature. The spectrum of original dextrin (Fig. 5a, bottom) shows the profile expected with basis on previous reports for starch-based polysaccharides [24-26]. The central band at $72 \mathrm{ppm}$ accommodates the overlapped contributions from C2, 3, 5 and ordered C4 environments [26]. Glycosidic carbons $\mathrm{C} 1$ and $\mathrm{C} 4$ profiles are most sensitive to chain conformations, the broad $81 \mathrm{ppm}$ peak arising from amorphous C4 environments. The $\mathrm{C} 1$ region, in original dextrin, comprises overlapped contributions from a predominating ordered single helical conformation ( $\mathrm{C} 1$ at $103 \mathrm{ppm}$ ) and a less abundant range of disordered conformations (C1 at 90-100 ppm) [25]. VA grafting leads to a
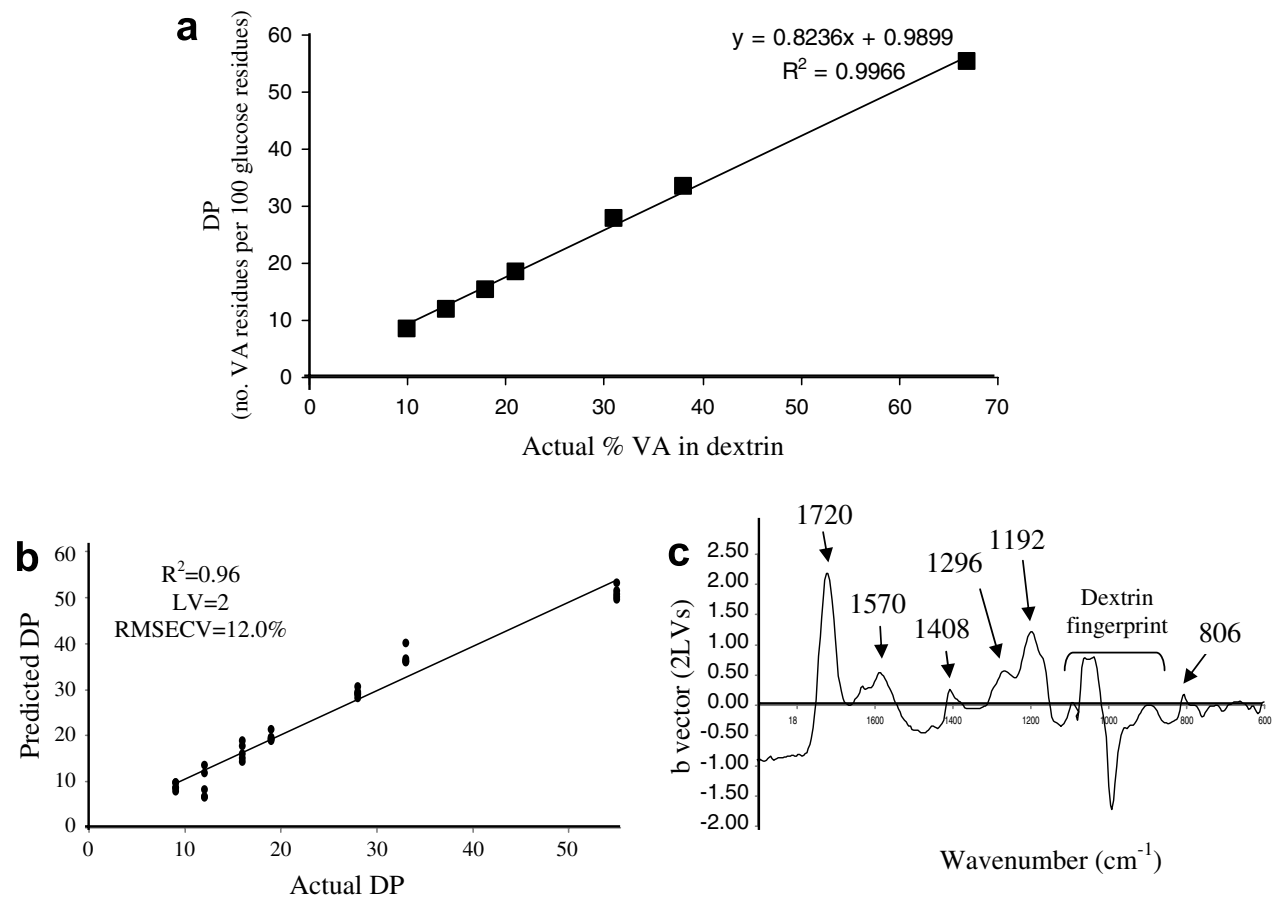

Wavenumber $\left(\mathrm{cm}^{-1}\right)$

Fig. 4. (a) Plot of the degree of polymerization, DP, calculated by FTIR integration, and \%VA of original dextrin sample; PLS/FTIR (b) relationship between actual and estimated DP values and (c) b vector for polymerized samples (hydrogels). 


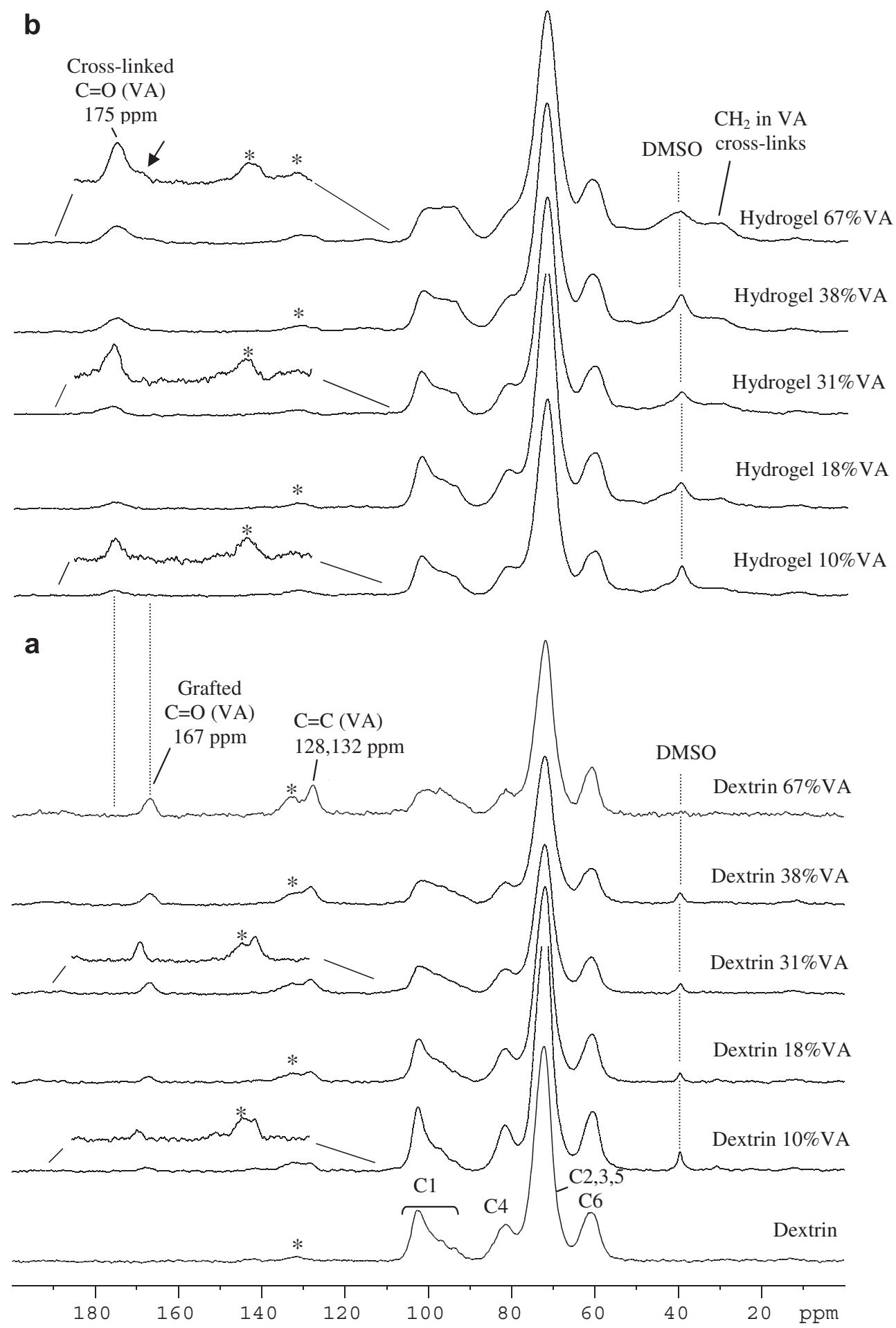

Fig. 5. ${ }^{13} \mathrm{C} \mathrm{CP} / \mathrm{MAS}$ spectra of (a) original dextrin and dextrin substituted with 10, 18, 31, 38 and 67\% VA; (b) corresponding polymerized samples (hydrogels). The main assignments are shown and the inserts show expansions of the carbonyl region. *: spinning sidebands.

carbonyl peak at $167 \mathrm{ppm}$ and contribution from $\mathrm{C}=\mathrm{C}$ carbons at 132 (superimposed with a spinning side band, Fig. 5a) and $128 \mathrm{ppm}$. Ungrafted VA carbonyls were seen to resonate at $163 \mathrm{ppm}$, so that the presence of this peak is useful to evaluate the efficiency of grafting. The signal at $40 \mathrm{ppm}$ arises from residual DMSO from the reactive medium, consistently with its random intensity variation within samples. Reaction with VA leads to significant broadening 
Table 2

Proton spin-lattice relaxation times $\left(T_{1 \mathrm{H}}\right)$, cross-polarization times $\left(T_{\mathrm{CH}}\right)$ and proton spin-lattice relaxation times in the rotating frame $\left(T_{1} \rho_{\mathrm{H}}\right)$ for three selected dextrin hydrogels

\begin{tabular}{|c|c|c|c|c|}
\hline \multirow[t]{3}{*}{ ppm } & \multirow[t]{3}{*}{ Assignment } & \multicolumn{3}{|c|}{ Hydrogel samples } \\
\hline & & Dextrin & Dextrin & Dextrin \\
\hline & & $21 \% \mathrm{VA}$ & $38 \% \mathrm{VA}$ & $67 \% \mathrm{VA}$ \\
\hline \multicolumn{5}{|l|}{$T_{1 \mathrm{H}} / \mathrm{s}$} \\
\hline 32 & $\mathrm{CH}_{2}$ VA polym. & n.d. & n.d. & n.d. \\
\hline 40 & $\mathrm{CH}_{3}$ (res.DMSO) & $0.931 \pm 0.121$ & $1.138 \pm 0.175$ & $1.154 \pm 0.164$ \\
\hline 61 & C6 glc. & $0.969 \pm 0.146$ & $1.251 \pm 0.171$ & $1.200 \pm 0.175$ \\
\hline 72 & C2,3,5 glc. & $0.922 \pm 0.134$ & $1.231 \pm 0.152$ & $1.201 \pm 0.188$ \\
\hline 81 & C4 glc. & $0.940 \pm 0.107$ & $1.241 \pm 0.195$ & $1.173 \pm 0.235$ \\
\hline 102 & C1 glc. & $0.871 \pm 0.126$ & n.d. & $1.182 \pm 0.192$ \\
\hline 128 & $\begin{array}{l}\mathrm{C}=\mathrm{C} \text { VA } \\
\text { unpolym. }\end{array}$ & $0.818 \pm 0.136$ & n.d. & $0.992 \pm 0.190$ \\
\hline 167 & $\begin{array}{l}\mathrm{C}=\mathrm{O} \text { VA } \\
\text { unpolym. }\end{array}$ & n.d. & n.d. & n.d. \\
\hline 175 & $\begin{array}{l}\mathrm{C}=\mathrm{O} \text { VA } \\
\text { polym. }\end{array}$ & n.d. & $\begin{array}{l}(1.176 \pm 0.272 \\
23 \%)\end{array}$ & $1.024 \pm 0.200$ \\
\hline \multicolumn{5}{|c|}{$T_{1 \rho H} / m s$} \\
\hline 32 & $\mathrm{CH}_{2}$ VA polym. & n.d. & n.d. & n.d. \\
\hline 40 & $\mathrm{CH}_{3}$ (res.DMSO) & $4.552 \pm 0.283$ & $4.704 \pm 0.408$ & $3.363 \pm 0.207$ \\
\hline 61 & C6 glc. & $2.471 \pm 0.190$ & $3.163 \pm 0.215$ & $2.547 \pm 0.074$ \\
\hline 72 & C2,3,5 glc. & $2.573 \pm 0.096$ & $\begin{array}{l}(4.317 \pm 1.490 \\
35 \%)\end{array}$ & $2.672 \pm 0.107$ \\
\hline 81 & C4 glc. & $2.401 \pm 0.085$ & $2.969 \pm 0.230$ & $2.817 \pm 0.118$ \\
\hline 102 & C1 glc. & $2.571 \pm 0.128$ & n.d. & $2.817 \pm 0.118$ \\
\hline 128 & $\begin{array}{l}\mathrm{C}=\mathrm{C} \text { VA } \\
\text { unpolym. }\end{array}$ & n.d. & n.d. & n.d. \\
\hline 167 & $\begin{array}{l}\mathrm{C}=\mathrm{O} \text { VA } \\
\text { unpolym. }\end{array}$ & n.d. & $6.722 \pm 1.038$ & n.d. \\
\hline 175 & $\begin{array}{l}\mathrm{C}=\mathrm{O} \text { VA } \\
\text { polym. }\end{array}$ & $3.536 \pm 0.141$ & $6.197 \pm 0.873$ & $2.05158 \pm 0.119$ \\
\hline \multicolumn{5}{|c|}{$T_{C H} / m s$} \\
\hline 32 & $\mathrm{CH}_{2}$ VA polym. & n.d. & n.d. & n.d. \\
\hline 40 & $\mathrm{CH}_{3}($ res.DMSO $)$ & $0.114 \pm 0.014$ & $0.114 \pm 0.015$ & $0.120 \pm 0.020$ \\
\hline 61 & C6 glc. & n.d. & $0.031 \pm 0.0033$ & $0.054 \pm 0.005$ \\
\hline 72 & C2,3,5 glc. & $0.041 \pm 0.003$ & $\begin{array}{l}(0.036 \pm 0.021 \\
58 \%)\end{array}$ & $0.080 \pm 0.008$ \\
\hline 81 & C4 glc. & $0.046 \pm 0.003$ & $0.069 \pm 0.007$ & $0.074 \pm 0.008$ \\
\hline 102 & C1 glc. & $0.041 \pm 0.004$ & n.d. & $0.074 \pm 0.008$ \\
\hline 128 & $\begin{array}{l}\mathrm{C}=\mathrm{C} \text { VA } \\
\text { unpolym. }\end{array}$ & n.d. & n.d. & n.d. \\
\hline 167 & $\begin{array}{l}\mathrm{C}=\mathrm{O} \text { VA } \\
\text { unpolym. }\end{array}$ & n.d. & $0.538 \pm 0.098$ & n.d. \\
\hline 175 & $\begin{array}{l}\mathrm{C}=\mathrm{O} \text { VA } \\
\text { polym. }\end{array}$ & n.d. & $1.066 \pm 0.213$ & n.d. \\
\hline
\end{tabular}

All values have uncertainties $<20 \%$, unless stated otherwise for values in brackets.

n.d.: Not determined due to weak or broad signal features.

of the C1 profile and, to a lesser extent, C4 profile (Fig. 5a), with increasing \%VA promoting an increase in amorphous polysaccharide (90-100 ppm) in detriment of the ordered single helical conformation (103 ppm), practically absent at $67 \%$ VA. This brings light into the nature of the structural changes affecting dextrin substituted with VA.

VA polymerization results in broadened spectra throughout (Fig. 5b) which shows that polymerization results in a further disordered system. The persistence of the broad $\mathrm{C} 4$ resonance at $81 \mathrm{ppm}$ confirms this, together with the $\mathrm{C} 1$ band which reflects the changes already noted for the unpolymerized samples. Cross-linked VA carbonyls resonate at lower field, $175 \mathrm{ppm}$, and a broad peak appears at $32 \mathrm{ppm}$, arising from VA methylene carbons at the crosslinks (Fig. 5b). In addition, the double bonds contribution at 132 and $128 \mathrm{ppm}$ becomes rather broad and weak, overlapping with spinning side bands corresponding to the $72 \mathrm{ppm}$ and the $\mathrm{C}=\mathrm{O}$ peaks (Fig. $5 \mathrm{~b}$ ). For the hydrogel containing higher VA amount, a shoulder at $167 \mathrm{ppm}$ is visible (noted with an arrow), reflecting the VA groups remaining unpolymerized.

In order to explore the possibility of using the ${ }^{13} \mathrm{C} \mathrm{CP} /$ MAS to quantify DS and DP in dextrin samples, a spin counting experiment was performed in order to establish the amount of carbon visible in the ${ }^{13} \mathrm{C} \mathrm{CP} / \mathrm{MAS}$ spectra. This is particularly important in natural materials that may contain paramagnetic impurities, which will enhance spin relaxation rates and lead to partial signal loss in the spectra. The procedure was based on that described previously for a range of natural compounds [14] and Table 3 shows that the percentage of carbon observability, $\% \mathrm{C}_{\mathrm{obs}}$, for selected hydrogels is in the $83-99 \%$ range, which is satisfactory, considering previous results [14]. Deviations from $100 \% \mathrm{C}_{\mathrm{obs}}$ occur if samples have long $T_{\mathrm{CH}}$ values and/or short proton $T_{1} \rho$ relaxation times and this may be corrected for taking into account the timescale of those parameters [14]. To evaluate the extent of improvement achievable for dextrin, the average $T_{1} \rho \mathrm{H}$ was measured for three hydrogels (with 21, 38 and 67\% VA), with improved precision on the decaying part of the CP curve. After correction of spectral areas with basis on $T_{1} \rho_{\mathrm{H}}$ (Table 3 ), the values of corrected $\% C_{\mathrm{obs}}$ showed some improvement for dextrin with 21\%VA but no significant improvements for the remaining samples, which in any case have high enough carbon observabilities. Therefore, if desired, some optimization may be achieved by performing $T_{1} \mathrm{\rho H}$-based corrections on dextrin ${ }^{13} \mathrm{C} \mathrm{CP} / \mathrm{MAS}$ spectra but, since $\% C_{\mathrm{obs}}$ are originally satisfactorily high and the correction procedure becomes unpractical for more than a few samples due to extended acquisition times, the uncorrected ${ }^{13} \mathrm{C}$ $\mathrm{CP} / \mathrm{MAS}$ spectra were hereafter employed in this work.

The possibility of direct quantitation of DS and DP, by integration of carbonyl ${ }^{13} \mathrm{C} \mathrm{CP} / \mathrm{MAS}$ resonances runs into several stumbling blocks, one being the weak and broad nature of those resonances (Fig. 5) and the other the fact that the quantitativity condition for ${ }^{13} \mathrm{C} \mathrm{CP} /$ MAS spectra $\left(T_{\mathrm{CH}}<<1 \mathrm{~ms}\right.$ contact time $\left.\ll T_{1 \rho \mathrm{H}}\right)$ is not satisfactorily met for all peaks. Indeed, $T_{1} \rho \mathrm{H}$ values of about $2-3 \mathrm{~ms}$ were found (Table 2), together with $T_{\mathrm{CH}}$ values of the order of ms for VA carbonyls, thus rendering direct peak integration not recommended unless signals correction is performed.

\section{Table 3}

NMR spin counting results for hydrogel samples and $T_{1} \rho^{-b}$-bed corrections for three of the samples

\begin{tabular}{lllll}
\hline Hydrogel sample & $\% \mathrm{C}^{\mathrm{a}}$ & $\begin{array}{l}\text { Uncorrected } \\
\% C_{\text {obs }}\end{array}$ & $T_{1 \rho \mathrm{H}} / \mathrm{ms}$ & $\begin{array}{l}\text { Corrected } \\
\% C_{\text {obs }}\end{array}$ \\
\hline Dextrin-VA 10\% & 34.92 & 94 & n.d. & n.d. \\
Dextrin-VA 14\% & 15.88 & 91 & n.d. & n.d. \\
Dextrin-VA 18\% & 23.28 & 93 & n.d. & n.d. \\
Dextrin-VA 21\% & 35.84 & 84 & $2.33 \pm 0.34$ & 94 \\
Dextrin-VA 31\% & 37.01 & 83 & n.d. & n.d. \\
Dextrin-VA 38\% & 37.14 & 89 & $3.17 \pm 0.37$ & 86 \\
Dextrin-VA 67\% & 38.19 & 99 & $2.61 \pm 0.44$ & 99 \\
\hline
\end{tabular}

n.d.: Not determined.

a Determined by elemental analysis. 
However, an alternative approach is the use of PLS regression of the CP/MAS spectra, using the known \%VA values as reference for unpolymerized samples, and the FTIR-based DP values as reference for polymerized samples, as will be discussed below. A closer look at the CP dynamics parameters in Table 2 shows that both $T_{1} \rho_{\mathrm{H}}$ and $T_{\mathrm{CH}}$ values are higher for the 40 ppm peak, which confirms its assignment to DMSO since it indicates that the compound is in a different spin reservoir than dextrin, rendering spin diffusion insufficient for complete $T_{1} \rho \mathrm{H}$ averaging. Efficient spin diffusion is seen for all dextrin resonances, but VA carbonyls are characterized by longer $T_{1 \rho \mathrm{H}}$ for 21 and $38 \% \mathrm{VA}$ hydrogels (Table 2), reflecting a different environment characterizing the cross links. Interestingly, for the $67 \%$ VA hydrogel carbonyl, $T_{1} \rho \mathrm{H}$ is closer to those of dextrin peaks, which is consistent with the higher density noted microscopically for this material (data not shown), the subject of an ongoing parallel work.

Regarding the use of ${ }^{13} \mathrm{C} \mathrm{CP} / \mathrm{MAS}$ spectra for DS and DP quantitation by PLS/NMR, Fig. 6a and b shows the actual \%VA vs. estimated \%VA plot and b vector obtained for unpolymerized samples. It is interesting to note that the relationship plot (Fig. 6a) is of improved quality throughout the whole \%VA range compared to those obtained by FTIR (Fig. 3b). A similar observation is noted for the polymerized samples (Fig. 6c), thus indicating that direct PLS analysis of the ${ }^{13} \mathrm{C} \mathrm{CP} / \mathrm{MAS}$ of these samples enables a better quantitation model to be built, relatively to PLS/FTIR. The PLS/NMR b vector profile for both unpolymerized and polymerized (Fig. $6 \mathrm{~b}$ and $\mathrm{d}$ ) show that VA carbonyl and double bonded carbon resonances are largely responsible for the spectral variations noted. However, large variations affect the dextrin resonances as well, particularly the $\mathrm{C} 1$ region (Fig. $6 \mathrm{~b})$. This reflects the large changes in dextrin $\mathrm{C} 1$ profile already noted, as a function of \%VA, with a narrow (negative) $\mathrm{C} 1$ peak at $103 \mathrm{ppm}$ (arising from ordered single helical conformation) predominating at low \%VA samples and a broad (positive) C1 band at 90$100 \mathrm{ppm}$ (reflecting a more amorphous system) predominating at the high \%VA samples. The fact that this is observed for both unpolymerized and polymerized samples (Figs. 5 and 6 )b-d indicates that these conformational changes are triggered mainly by increasing VA content, rather than by polymerization. This result expresses the advantages of NMR in helping to identify the structural changes affecting dextrin and of PLS/NMR as a rapid quantitation method for DS and DP, avoiding lengthy area correction methods for discrete NMR peak integration.

\subsection{D-COS between FTIR and NMR data}

2D-COS correlation between FTIR and NMR data may be of use to detect, either direct (or positive) simultaneous changes in both datasets (and thus confirm spectral assignments or detect direct inter-compound effects), or inverse (or negative) simultaneous changes (and thus detect inverse inter-compound effects). The synchronous map obtained by 2D correlation between FTIR and NMR data for the unpolymerized dextrin samples (Fig. 7a) shows that the $\mathrm{C} 1$ peak at $103 \mathrm{ppm}$ in NMR correlates directly (red spot) with a shoulder at the lower wavenumber side $\left(995 \mathrm{~cm}^{-1}\right.$ ) of the $1000 \mathrm{~cm}^{-1}$ band, which is the main dextrin band in the fingerprint region (Fig. 2a, bottom). This shows that $\mathrm{C} 1$ vibrations contribute significantly for that FTIR dextrin band, the changes of which may be interpreted as chain conformational changes affecting glycosidic bonds. This also brings new light into the assignment of the negative ca. $1000 \mathrm{~cm}^{-1}$ peak in the FTIR b vector profile in Fig. $3 c$ which, thus, indicates conformation changes at glycosidic linkages. The same $\mathrm{C} 1$ peak
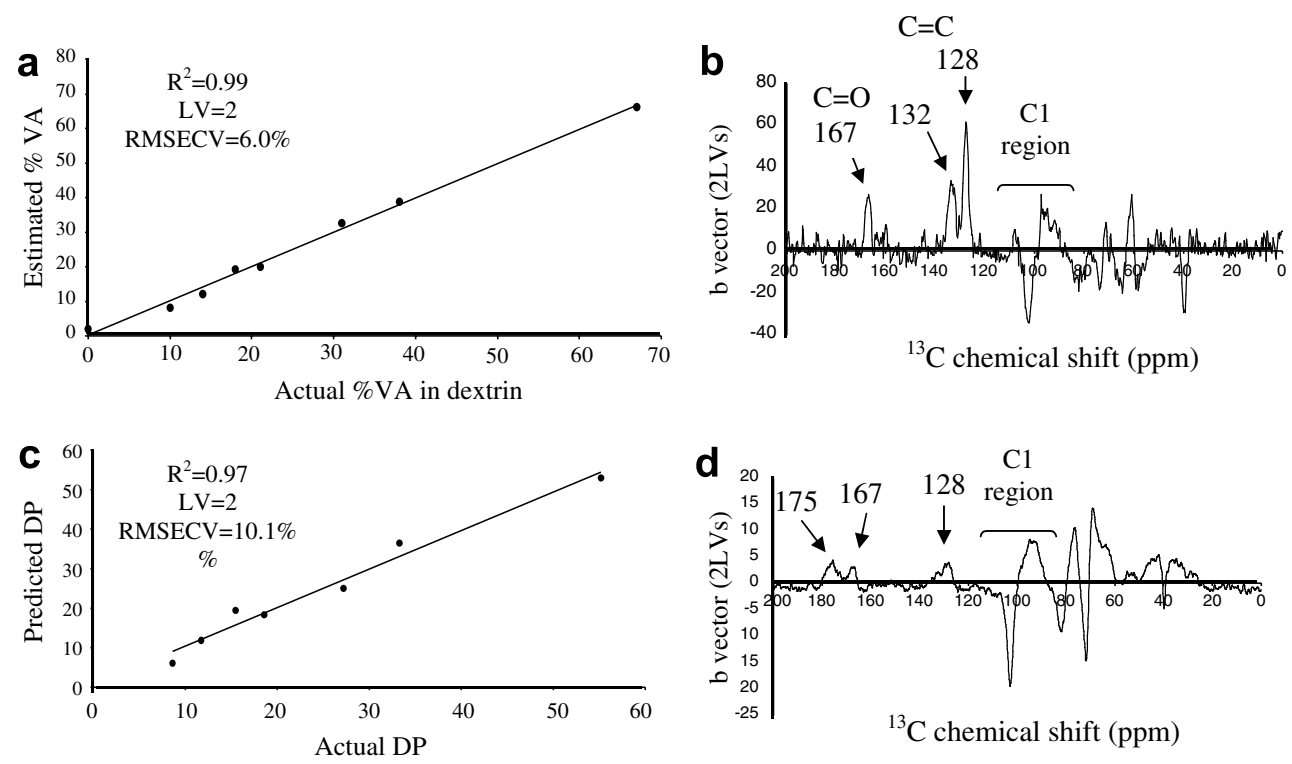

Fig. 6. PLS/NMR: (a) relationship between actual $v$ s. estimated \%VA values (b) b vector profile obtained for unpolymerized dextrin samples, as a function of \%VA as determined by ${ }^{1} \mathrm{H}$ NMR spectroscopy and PLS/NMR (c) relationship between actual vs. estimated DP values and (d) b vector profile obtained for polymerized dextrin samples, as a function of DP as determined by FTIR spectroscopy. 

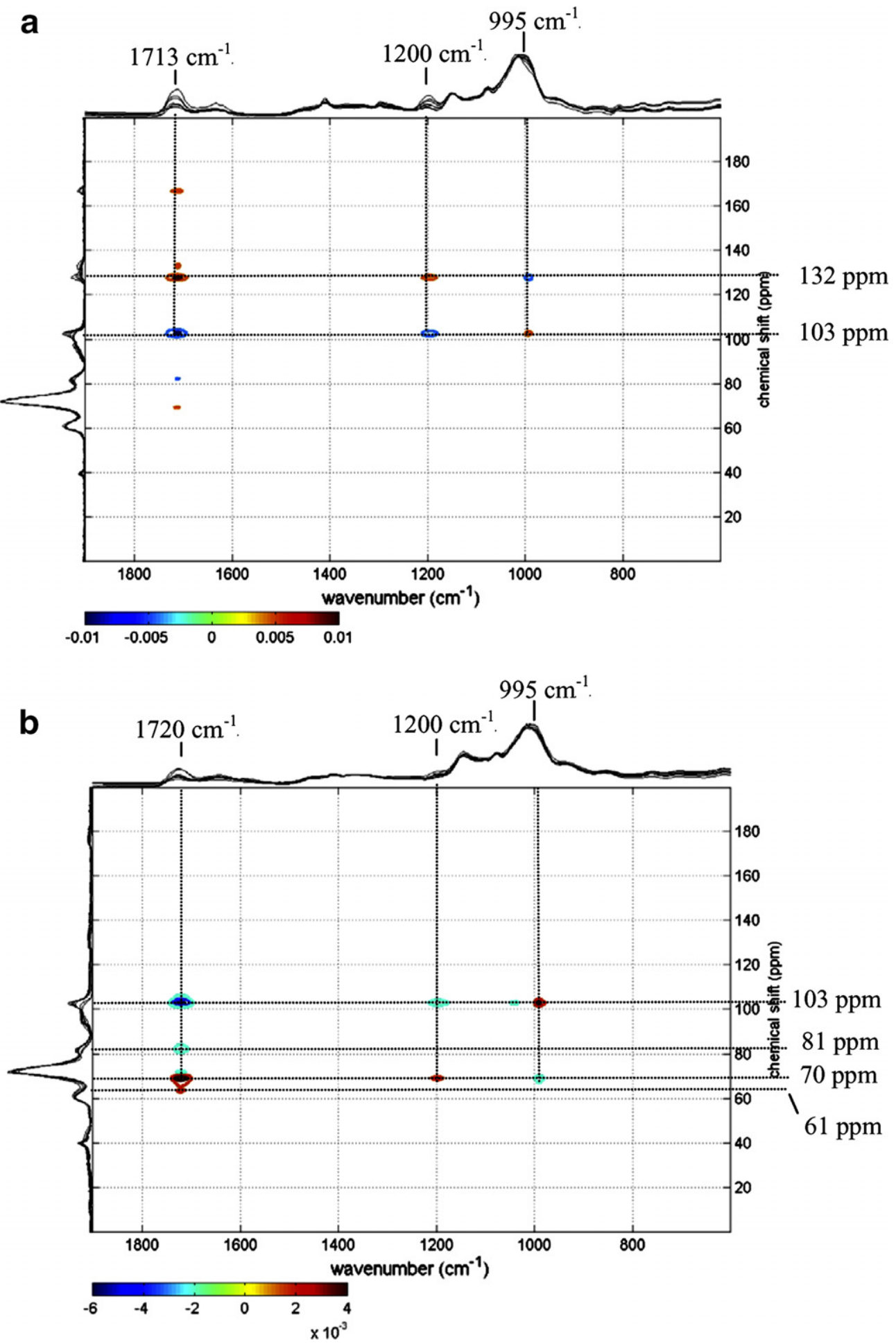

Fig. 7. 2D-COS synchronous maps of (a) unpolymerized dextrin and (b) polymerized dextrin.

correlates inversely (blue spots) with FTIR bands at ca. 1200 and $1713 \mathrm{~cm}^{-1}$. These are VA bands and this inverse correlation confirms that, as \%VA increases, the more ordered chain conformation (with $\mathrm{C} 1$ at $103 \mathrm{ppm}$ ) decreases in content. The direct correlations between peaks at ca.
$130 \mathrm{ppm}$ ( $\mathrm{C}=\mathrm{C}$ of unpolymerized VA) with VA bands at $1713 \mathrm{~cm}^{-1}$ and $1200 \mathrm{~cm}^{-1}$, and peak at $167 \mathrm{ppm}(\mathrm{C}=\mathrm{O}$ of unpolymerized VA) with VA band at $1713 \mathrm{~cm}^{-1}$ band are expected since all bands arise from VA moieties and, thus, vary directly with each other. 
Regarding the 2D synchronous map obtained for the polymerized samples (Fig. 7b), only correlations to dextrin NMR bands are observed due to the significant broadening of VA bands in the NMR dimension. The correlations observed for the $\mathrm{C} 1$ at $103 \mathrm{ppm}$ are similar to those noted above for unpolymerized samples, confirming the direct contribution of glycosidic bonds at $995 \mathrm{~cm}^{-1}$ in FTIR and the inverse correlation of the ordered polysaccharide conformation with VA content. Interestingly, additional stronger correlations are noted between the FTIR dimension and the NMR ring resonances, indicating that polymerization has an important effect on glucose ring conformation, compared to the effects of grafting (Fig. 7a) which seem to hardly affect ring conformations. An NMR signal at the high ppm side of the central peak at $72 \mathrm{ppm}$ (70 ppm) correlates strongly and directly with VA bands at $1720 \mathrm{~cm}^{-1}$ and $1200 \mathrm{~cm}^{-1}$, and inversely with the $995 \mathrm{~cm}^{-1} \mathrm{C} 1$-related band. This shows that, as \%VA increase in the hydrogels, dextrin conformation is changed, not only at the glycosidic bands $\left(995 \mathrm{~cm}^{-1}\right.$ and $103 \mathrm{ppm}$ ) but also at the glucose ring, affecting a resonance at $70 \mathrm{ppm}$, buried under the broad $72 \mathrm{ppm}$ dextrin peak. Weaker correlations are visible also for C6 (61 ppm) and C4-amorphous (81 ppm), indicating that these carbons are also slightly sensitive to the effect of polymerization and \%VA in the sample.

2D-COS analyses allow, therefore, important relationships between FTIR and NMR data to be identified and further, initially unresolved, structural information to be obtained, as a function of the DS or DP. The asynchronous maps are not shown here due to their low significance (variations within the rounding-off error), suggesting that the behavior observed for this system is mainly characterized by linear relationships.

\section{Conclusions}

FTIR spectroscopy and ${ }^{13} \mathrm{C} C \mathrm{C} / \mathrm{MAS}$ NMR spectroscopy have been applied to the study of dextrin structural changes, upon hydrogel formation by vinyl acrylate (VA) grafting followed by free radical polymerization. DS and DP have been satisfactorily evaluated up to 40\%VA by both measurement of the FTIR $I_{806} / I_{760}$ ratio and partial least squares-PLS FTIR, the latter being a faster method which avoids errors related to baseline correction and intensity measurements. Above 40\%VA, DS and DP are underestimated by both FTIR-based methods, however, the use of ${ }^{13} \mathrm{C} \mathrm{CP/MAS} \mathrm{NMR} \mathrm{leads} \mathrm{to} \mathrm{significantly} \mathrm{improved} \mathrm{PLS/}$ NMR models for DS and DP determination in all the \%VA range studied. The PLS/NMR method is recommended, relatively to the direct integration methods because the latter is seriously hindered by the broad nature of VA peaks and the need for area correction, due to their CP dynamics.

Both FTIR and NMR methods show that, as the \%VA increases, structural changes occur in the polysaccharide. Changes observed in C1 and C4 NMR profiles of unpolymerized and polymerized samples have shown that an ordered single helical conformation predominates for low \%VA samples, being replaced by a disordered system as \%VA increases. In addition, CP dynamics measurements indicate that a more dense system is formed at the highest
\%VA (67\%). Finally, changes observed by NMR have been correlated to the FTIR spectra by a 2D-COS method, enabling the identification of conformation-sensitive bands in FTIR spectra and indicating that ring conformations are particularly affected by polymerization.

\section{Acknowledgements}

Funding is acknowledged from the Foundation for Science and Technology, Portugal, for project POCI/QUI/ $56229 / 2004$ and grants for H.G. and C.G. (SFRH/22242/ 2005). The authors also wish to acknowledge CICECO for partial funding of this work.

\section{References}

[1] Peppas NA, Bures P, Leobandung W, Ichikawa H. Hydrogels in pharmaceutical formulations. Eur J Pharm Biopharm 2000;50: 27-46.

[2] Peppas NA. Hydrogels. Biomaterials science: an introduction to materials in medicine. San Diego: Academic Press; 1996. p. 60-4.

[3] McCulloch I, Shalaby SW. Tailored polymeric materials for controlled delivery systems. Washington DC: American Chemical Society; 1998.

[4] Kane JB, Tompkins RG, Yarmush ML, Burke JF. Burn dressings. Biomaterials science: an introduction to materials in medicine. San Diego: Academic Press; 1996. p. 360-70.

[5] Drury JL, Mooney DJ. Hydrogels for tissue engineering: scaffold design variables and applications. Biomaterials 2003;24(24): 4337-51.

[6] Wong WH, Mooney DJ. Synthesis and properties of biodegradable polymers used as synthetic matrices for tissue engineering. Synthetic biodegradable polymer scaffolds. Berlin: Springer; 1997. p. 51-82.

[7] Marques AP, Reis RL, Hunt JA. The biocompatibility of novel starchbased polymers and composites: in vitro studies. Biomaterials 2002;23(6):1471-8.

[8] Ferreira L, Carvalho R, Gil MH, Dordick JS. Enzymatic synthesis of inulin-containing hydrogels. Biomacromolecules 2002;3(2): 333-41.

[9] Ferreira L, Gil MH, Dordick JS. Enzymatic synthesis of dextrancontaining hydrogels. Biomaterials 2002;23(19):3957-67.

[10] Zhang LM, Yang C, Yan L. Perspectives on: strategies to fabricate starch-based hydrogels with potential biomedical applications. J Bioact Compat Polym 2005;20(3):297-314.

[11] Carvalho JM, Gonçalves C, Gil AM, Gama FM. Production and characterisation of a new dextrin based hydrogel. Eur Polym J 2007;43(7):3050-9.

[12] Vervoort L, Van den Mooter G, Augustijns P, Busson R, Toppet S, Kinget R. Inulin hydrogels as carriers for colonic drug targeting: I synthesis and characterization of methacrylated inulin and hydrogel formation. Pharm Res 1997;14(12):1730-7.

[13] Gonçalves C, Martins JA, Gama FM. Self-assembled nanoparticles of dextrin substituted with hexadecanethiol. Biomacromolecules 2007;8(2):392-8.

[14] Yan L, Oades JM. The use of spin counting for determining quantitation in solid state C-13 NMR spectra of natural organic matter 1 . Model systems and the effects of paramagnetic impurities. Geoderma 2000;96(1-2):101-29.

[15] Helland IS. Some theoretical aspects of partial least squares regression. Chemom Intell Lab 2001;58(2):97-107.

[16] Martens H. Reliable and relevant modelling of real world data: a personal account of the development of PLS Regression. Chemom Intell Lab 2001;58(2):85-95.

[17] Wold S, Sjöström M, Eriksson L. PLS-regression: a basic tool of chemometrics. Chemom Intell Lab 2001;58(2):109-30.

[18] Wold S, Martens H, Wold H. Lecture notes in mathematics 1983. In: Ruhe A, Kägström B, editors. Proceedings of the conference matrix pencils. Heidelber: Springer-Verlag; 1983. p. 286-93.

[19] Noda I. Two-dimensional infrared spectroscopy. J Am Chem Soc 1989;111:8116-8.

[20] Noda I. Two-dimensional infrared (2D IR) spectroscopy - theory and applications. Appl Spectrosc 1990;44(4):550-61.

[21] Noda I, Dowrey AE, Marcott C, Story GM, Ozaki Y. Generalized twodimensional correlation spectroscopy. Appl Spectrosc 2000;54(7): 236A-48A. 
[22] Noda I, Downrey AE, Marcott C. Recent developments in 2dimensional infrared (2D-IR) correlation spectroscopy. Appl Spectrosc 1993;47(9):1317-23.

[23] Lefèvre T, Arseneault K, Pézolet M. Study of protein aggregation using two-dimensional correlation infrared spectroscopy and spectral simulations. Biopolymers 2004;73(6):705-15.

[24] Gidley MJ, Bociek SM. C-13 CP/MAS NMR-studies of amylose inclusion complexes, cyclodextrins, and the amorphous phase of starch granules - relationships between glycosidic linkage conformation and solid state C-13 chemical shifts. J Am Chem Soc 1988;110(12):3820-9.

[25] Shefer A, Shefer S, Kost J, Langer R. Structural characterization of starch networks in the solid state by cross-polarization magic-anglespinning C-13 NMR-spectroscopy and wide angle x-ray diffraction. Macromolecules 1992;25(25):6756-60.

[26] Tang HR, Hills BP. Use of C-13 MAS NMR to study domain structure and dynamics of polysaccharides in the native starch granules. Biomacromolecules 2003;4(5):1269-76. 